\title{
Drift-Induced Step Instabilities Due to the Gap in the Diffusion Coefficient
}

\author{
Masahide Sato * Makio $\mathrm{Uwaha}^{a}$, and Yukio Saito ${ }^{b}$ \\ Information Media Center of Kanazawa University, Kakuma-cho, Kanazawa 920-1192, Japan \\ ${ }^{a}$ Department of Physics, Nagoya University, Furo-cho, Chikusa-ku, Nagoya 464-8602, Japan \\ ${ }^{b}$ Department of Physics, Keio University, 3-14-1 Hiyoshi, Kohoku-ku, Yokohama 223-8522, Japan
}

(Dated: November 18, 2018)

\begin{abstract}
On a $\mathrm{Si}(111)$ vicinal face near the structural transition temperature, the $1 \times 1$ structure and the $7 \times 7$ structure coexist in a terrace: the $1 \times 1$ structure is in the lower side of the step edge and the $7 \times 7$ structure in the upper side. The diffusion coefficient of adatoms is different in the two structures. Taking account of the gap in the diffusion coefficient at the step, we study the possibility of step wandering induced by drift of adatoms. A linear stability analysis shows that the step wandering always occurs with step-down drift if the diffusion coefficient has a gap at the step. Formation of straight grooves by the step wandering is expected from a nonlinear analysis. The stability analysis also shows that step bunching occurs irrespective of the drift direction if the diffusion in the lower side of the step is faster. The step bunching disturbs the formation of grooves. If step-step repulsion is strong, however, the step bunching is suppressed and the straight grooves appear. Monte Carlo simulation confirms these predictions.
\end{abstract}

PACS numbers: $81.10 . \mathrm{Aj}, 05.70 . \mathrm{Ln}, 47.20 . \mathrm{Hw}, 68.35 . \mathrm{Fx}$

\section{INTRODUCTION}

On vicinal faces of $\mathrm{Si}(111)$ [1, 2, 3] and $\mathrm{Si}(001)$ [4], step wandering occurs at high temperatures when a specimen is heated by direct electric current. The current direction to cause the step wandering is step-down on the $\mathrm{Si}(111)$ vicinal face [1, 2, 3] and step-up on the $\operatorname{Si}(001)$ vicinal face [4].

The cause of the step wandering is drift of adatoms by the current [5, 6, 7, 8]. The drift is in the same direction as the current [6, 7]. If the step is impermeable [9, 10], the step wandering occurs with step-down drift, as in the $\mathrm{Si}(111)$ vicinal face [1, 2, 3]. If there is alternation of the anisotropy in the diffusion coefficient on consecutive terraces 11], as in the $\mathrm{Si}(001)$ vicinal face [4] the step wandering occurs with step-up drift.

On $\mathrm{Si}(111)$ surfaces, the $1 \times 1$ structure is reconstructed and the $7 \times 7$ structure appears at low temperatures $\left(\leq 860^{\circ} \mathrm{C}\right)$. In a vicinal face near the transition temperature, the $7 \times 7$ structure spreads from the upper side of the steps, and the two structures coexist in a terrace. Recently, Hibino and co-workers 12] observed step wandering near $860^{\circ} \mathrm{C}$ during growth. Due to the in-phase step wandering, grooves perpendicular to the steps appear on the vicinal face. Kato and co-workers 13 studied the step wandering theoretically. Focusing on the difference in diffusion coefficient of the two structures, they showed that the step wandering occurs in growth if the diffusion coefficient on the $1 \times 1$ structure is larger than that on the $7 \times 7$ structure.

With the two phases coexisting, the drift of adatoms may also cause the step wandering instability on the $\mathrm{Si}(111)$ vicinal face. In this paper, we study the pos-

*Electronic address: sato@cs.s.kanazawa-u.ac.jp, Fax +81-76-234-6912 sibility of morphological instabilities induced by the drift of adatoms with the gap in the diffusion coefficient on the upper and the lower terraces.

\section{MODEL}

We consider a vicinal face where steps are running parallel to the $x$-direction bordering terraces of a width $l$ on average. The $y$-direction is chosen toward the step-down direction. If impingement and evaporation of adatoms are neglected, the adatom density $c(\boldsymbol{r}, t)$ is determined by

$$
\frac{\partial c(\boldsymbol{r}, t)}{\partial t}=\nabla \cdot \boldsymbol{j}(\boldsymbol{r}, t)
$$

where $\boldsymbol{j}(\boldsymbol{r}, t)$ is the adatom current on the surface. With step-down drift, the adatom current is given by

$$
\boldsymbol{j}(\boldsymbol{r}, t)=-D_{\mathrm{s}}(\boldsymbol{r})\left(\boldsymbol{\nabla} \boldsymbol{c}(\boldsymbol{r}, t)-\frac{F c(\boldsymbol{r}, t)}{k_{\mathrm{B}} T} \hat{\boldsymbol{e}}_{y}\right),
$$

where $D_{\mathrm{s}}(\boldsymbol{r})$ is the local diffusion coefficient, $F$ the force to cause the drift and is positive for the step-down drift, and $\hat{e}_{y}$ the unit vector toward the step-down direction. We assume that the diffusion coefficient $D_{\mathrm{s}}$ takes two values in a terrace: $D_{\mathrm{s}}=D_{1}$ in the lower side of a step edge, $y_{n}<y<y_{n}+l_{1}^{(n)}$, and $D_{\mathrm{s}}=D_{2}$ in the upper side, $y_{n-1}+l_{1}^{(n-1)}<y<y_{n}$, where $y_{n}(x, t)$ is the position of the $n$th step and $l_{1}^{(n)}(x, t)$ is the terrace width of the lower side structure $(1 \times 1$ in $\operatorname{Si}(111))$.

Solidification and melting occur at step edges. In local equilibrium at a step, the adatom density is given by

$$
\left.c\right|_{y_{n}}=c_{\mathrm{eq}}^{0}\left(1+\frac{\Omega \tilde{\beta}}{k_{\mathrm{B}} T} \kappa+\frac{\Omega}{k_{\mathrm{B}} T} \frac{\partial U_{n}}{\partial y_{n}}\right),
$$


where $c_{\mathrm{eq}}^{0}$ is the equilibrium adatom density at the isolated step, $\Omega$ the atomic area, $\tilde{\beta}$ the step stiffness, $\kappa$ the step curvature and $U_{n}$ the step-step interaction potential.

From the continuity of the adatom current and the adatom density, the boundary conditions at the phase boundary are given by

$$
\begin{aligned}
\left.\hat{\boldsymbol{n}}_{\mathrm{b}} \cdot \boldsymbol{j}\right|_{\left(y_{n}+l_{1}^{(n)}\right)+} & =\left.\hat{\boldsymbol{n}}_{\mathrm{b}} \cdot \boldsymbol{j}\right|_{\left(y_{n}+l_{1}^{(n)}\right)-}, \\
\left.c\right|_{\left(y_{n}+l_{1}^{(n)}\right)+} & =\left.c\right|_{\left(y_{n}+l_{1}^{(n)}\right)-},
\end{aligned}
$$

where $\hat{\boldsymbol{n}}_{\mathrm{b}}$ is the normal vector of the boundary and $+(-)$ indicates the lower (upper) side of the step.

By solving the diffusion equation (10) in a static approximation with the boundary conditions, Eqs. (31)-(5), the adatom density is determined. The normal step velocity $V_{n}$ is given by

$$
V_{n}=\Omega \hat{\boldsymbol{n}}_{\mathrm{s}} \cdot\left(\left.\boldsymbol{j}\right|_{y_{n}-}-\left.\boldsymbol{j}\right|_{y_{n}+}\right),
$$

where $\hat{\boldsymbol{n}}_{\mathrm{s}}$ is the normal vector of the step.

\section{STABILITY ANALYSIS}

When the steps and the boundaries of two phases are straight, the adatom density in the quasi-static approximation is given by $c(y)=A_{0}+B_{0} e^{f\left(y-y_{n}\right)}$, where $f=F / k_{\mathrm{B}} T$. In the lower side of a step, the coefficients $A_{0}$ and $B_{0}$ are given by

$$
\begin{aligned}
& A_{0}=\frac{D_{2} c_{\mathrm{eq}}^{0}\left(e^{f l^{(n)}}-1\right)}{D_{2} e^{f l_{2}}\left(e^{f l_{1}^{(n)}}-1\right)+D_{1}\left(e^{f l_{2}^{(n)}}-1\right)}, \\
& B_{0}=\frac{\left(D_{1}-D_{2}\right) c_{\mathrm{eq}}^{0}\left(e^{f l_{2}^{(n)}}-1\right)}{D_{2} e^{f l_{2}^{(n)}}\left(e^{f l_{1}^{(n)}}-1\right)+D_{1}\left(e^{f l_{2}^{(n)}}-1\right)},
\end{aligned}
$$

where $l^{(n)}=y_{n+1}-y_{n}$ and $l_{2}^{(n)}=l^{(n)}-l_{1}^{(n)}$. In the upper side of the step, the coefficients are given by the same form with the replacement $D_{1} \leftrightarrow D_{2}$ and $l_{1}^{(n)} \leftrightarrow l_{2}^{(n)}$. The adatom current $j_{0}$ is constant on the whole of the $n$-th terrace and is given by

$$
j_{0}\left(l^{(n)}\right)=\frac{D_{1} D_{2} c_{\mathrm{eq}}^{0} f\left(e^{f l^{(n)}}-1\right)}{D_{2} e^{f l_{2}^{(n)}}\left(e^{f l_{1}^{(n)}}-1\right)+D_{1}\left(e^{f l_{2}^{(n)}}-1\right)} .
$$

Since we have neglected the step repulsion, the current is a function of $l_{1}^{(n)}$ and $l_{2}^{(n)}$ and does not depend on the neighboring terrace widths.

If the ratio of the width of the two structures $\gamma=$ $l_{2}^{(n)} / l_{1}^{(n)}$ is fixed, Eq. (92) gives the current $j_{0}(l)$ as a function of the terrace width. When the steps are equidistant, the adatom current at the step positions from the upper terrace equals to that onto the lower terrace, $\left.j_{0}\right|_{y_{n}-}=\left.j_{0}\right|_{y_{n}+}$. The velocity of the steps vanishes and the steps do not move. If the step interaction is neglected, the stability of the equidistant steps for step pairing is determined by $j^{\prime}(l)$ since it controls the balance of the incoming and outgoing current with a pairing fluctuation of the terrace width (the repulsive interaction tends to stabilize the system). It is unstable for step pairing if $j_{0}^{\prime}(l)>0$ and stable otherwise. From Eq. (9), $j_{0}^{\prime}(l)$ is given by

$$
\begin{aligned}
j_{0}^{\prime}(l)= & \frac{\left(D_{1}-D_{2}\right) D_{1} D_{2} c_{\mathrm{eq}}^{0} f^{2} e^{f l_{2}} l_{1} l}{l\left[D_{2} e^{f l_{2}}\left(e^{f l_{1}}-1\right)+D_{1}\left(e^{f l_{2}}-1\right)\right]^{2}} \\
& \times\left(\frac{e^{f l}-1}{l}-\frac{e^{f l_{1}}-1}{l_{1}}\right) .
\end{aligned}
$$

When the surface diffusion in the lower side of the step is faster than that in the upper side $\left(D_{1}>D_{2}\right)$, the vicinal face is unstable for the step pairing. The stability is independent of the ratio $\gamma$ of the widths and the drift direction.

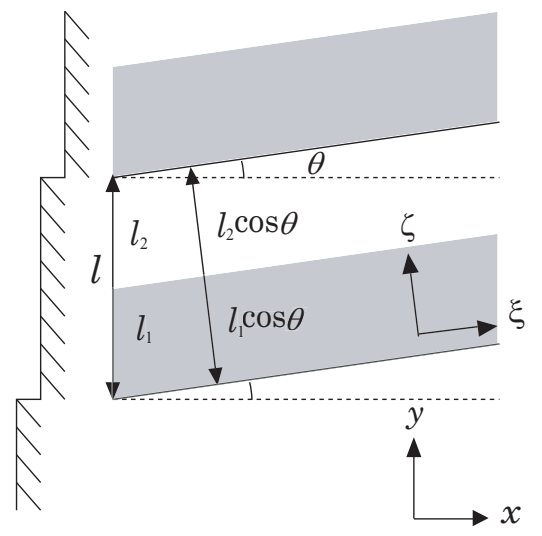

FIG. 1: A terrace bounded by tilted steps with an angle $\theta$.

To find the condition for the in-phase step wandering, we consider an equidistant train of straight steps tilted with an angle $\theta$ (Fig. 11). The adatom current on the terrace in the $x$-direction, $J_{x}^{(0)}$ is calculated as

$$
\begin{aligned}
J_{x}^{(0)}= & \int_{y_{n}}^{y_{n+1}} d y\left(j_{\|} \cos \theta-j_{\perp} \sin \theta\right) \\
= & \frac{\left(D_{1}-D_{2} e^{f_{\perp} l_{1 \perp}}\right)\left(e^{f_{\perp} l_{1 \perp}}-1\right)\left(e^{f_{\perp} l_{2}}-1\right)}{D_{2} e^{f l_{2}}\left(e^{f_{\perp} l_{1}}-1\right)+D_{1}\left(e^{f_{\perp} l_{2 \perp}}-1\right)} \\
& \times\left(D_{1}-D_{2}\right) c_{\mathrm{eq}}^{0} \tan \theta
\end{aligned}
$$

where $j_{\|}$and $j_{\perp}$ indicate the adatom current in the $\xi$ and $\zeta$ directions, $f_{\perp}=f \cos \theta, l_{\perp}=l \cos \theta, l_{1 \perp}=l_{1} \cos \theta$ and $l_{2 \perp}=l_{2} \cos \theta$. When the step distance is small enough, $f_{\perp} l_{\perp} \ll 1$, it becomes

$$
J_{x}^{(0)}=\frac{\left(D_{1}-D_{2}\right)^{2} f l_{1} l_{2} c_{\mathrm{eq}}^{0}}{\left(D_{1} l_{1}+D_{2} l_{2}\right)} \frac{\eta_{x}}{1+\left(\eta_{x}\right)^{2}} .
$$

where we assume $y_{n}(x, t)=n l+\eta(x, t)$ and $\eta_{x}=\partial \eta / \partial x$. In addition to Eq. (12), there is current $J_{x}^{(1)}$ caused by the change of the chemical potential along the step:

$$
J_{x}^{(1)}=-\cos ^{2} \theta\left(D_{1} l_{1 \perp}+D_{2} l_{2 \perp}\right) c_{\mathrm{eq}}^{0} \frac{\partial}{\partial x}\left(\frac{\mu}{k_{\mathrm{B}} T}\right),
$$


where $\mu=\Omega \tilde{\beta} \kappa$.

The evolution of the step position is determined by the adatom current in the $x$-direction as

$$
\begin{aligned}
\frac{\partial \eta}{\partial t} & =-\Omega \frac{\partial\left(J_{x}^{(0)}+J_{x}^{(1)}\right)}{\partial x} \\
& =-\frac{\partial}{\partial x}\left[\frac{\alpha_{2} \eta_{x}}{1+\eta_{x}^{2}}+\frac{\alpha_{4}}{1+\eta_{x}^{2}} \frac{\partial}{\partial x}\left(\frac{\eta_{x x}}{\left(1+\eta_{x}^{2}\right)^{3 / 2}}\right)\right]
\end{aligned}
$$

where the coefficients $\alpha_{2}$ and $\alpha_{4}$ are

$$
\begin{aligned}
& \alpha_{2}=\Omega \frac{\left(D_{1}-D_{2}\right)^{2} f l_{1} l_{2} c_{\mathrm{eq}}^{0}}{\left(D_{1} l_{2}+D_{2} l_{1}\right)}, \\
& \alpha_{4}=\Omega\left(D_{1} l_{1}+D_{2} l_{2}\right) c_{\mathrm{eq}}^{0} \frac{\Omega \tilde{\beta}}{k_{\mathrm{B}} T} .
\end{aligned}
$$

If the step position is of the form $\eta(x)=\eta_{0} e^{i q x+\omega_{q} t}$, the linear amplification rate is

$$
\omega_{q}=\alpha_{2} q^{2}-\alpha_{4} q^{4}
$$

The coefficient $\alpha_{4}$ is always positive and suppresses the step fluctuation. With step-up drift $(f<0)$ the coefficient $\alpha_{2}$ is negative and suppresses the step fluctuation, while $\alpha_{2}$ becomes positive and the step wandering occurs with step-down drift $(f>0)$.

Equation (14) is the same type of equation describing the step wandering in other conserved systems 13, 14, 15, 16]. The solution of the equation shows a regular periodic pattern whose amplitude increases in a power law of time as $t^{1 / 2}[14]$. As a result periodic grooves will be formed.

\section{MONTE CARLO SIMULATION}

We perform Monte Carlo simulation for solid-on-solid steps of a square lattice model. The boundary condition is helical in the $y$-direction and periodic in the $x$ direction. We assume that $\gamma$ is fixed to 1 so that the phase boundary is at $\left(y_{n}+y_{n+1}\right) / 2$ when the steps move. In the lower side of a step (supposedly the $1 \times 1$ region), an adatom on the site $(i, j)$ moves to $(i \pm 1, j)$ with the probability $p_{\mathrm{d}}=D_{1} / 4$ and to $(i, j \pm 1)$ with the probability $p_{\mathrm{d}}=D_{1}(1 \pm f a / 2) / 4$. In the upper side of a step (the $7 \times 7$ region), the parameter $D_{1}$ is replaced by $D_{2}$. The diffusion across the boundary of the two regions takes $D_{2}$. In our simulations, we assume that $\left(D_{1}, D_{2}\right)=(1, \alpha)$ or $(\alpha, 1)$ with $\alpha<1$.

Solidification and melting occur at the lower edge of the step positions [17]. The probabilities for solidification $p_{+}$and melting $p_{-}$are given by

$$
p_{ \pm}=\left[1+\exp \left(\frac{\Delta E_{\mathrm{s}}+\Delta U \mp \phi}{k_{\mathrm{B}} T}\right)\right]^{-1}
$$

where $\Delta E_{\mathrm{S}}$ is the increment of the step energy, $\phi$ the potential gain by solidification and $\Delta U$ is the change of the step-step interaction potential. We assume the repulsive interaction potential $U_{n}$ of the $n$th step takes the form

$$
U_{n}=\sum_{m=n \pm 1} \frac{A}{\left[y_{n}\left(x_{i}\right)-y_{m}\left(x_{i}\right)\right]^{2}},
$$

where $y_{n}\left(x_{i}\right)$ is the position of the $n$th step at $x=x_{i}$.

We first carry out the simulation with the diffusion coefficients $\left(D_{1}, D_{2}\right)=(1,0.1)$, the stiffness $\tilde{\beta} / k_{\mathrm{B}} T=$ 1.64 and the equilibrium density $c_{\mathrm{eq}}^{0}=0.18$. The system size is $512 \times 512$ and the initial step distance is $l=16$ (the step number is $N=32$ ). Initially, the steps are straight and equidistant, and there are a few adatoms on the terraces.

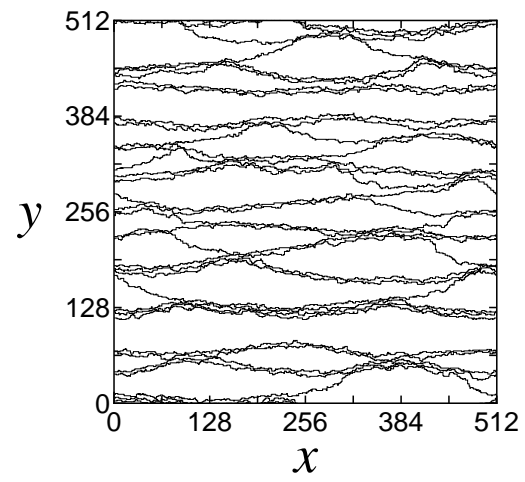

(a)

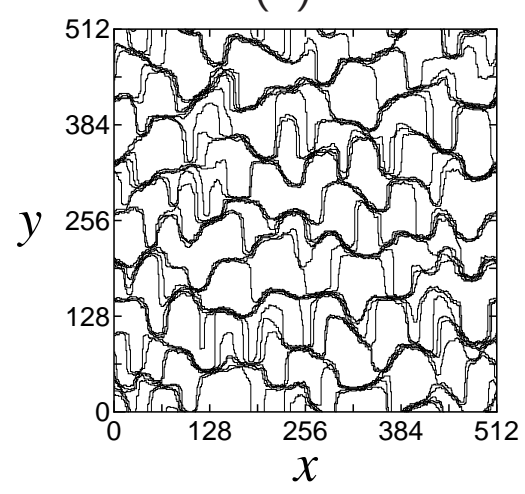

(b)

FIG. 2: Snapshots of a destabilized vicinal face for $D_{1}>D_{2}$ : with (a) step-up drift and (b) step-down drift .

When we neglect the step-step repulsive interaction, the vicinal face is unstable for the step bunching (Fig. 2(a)), which agrees with the analysis in Sec. III The step bunching occurs irrespective of the drift direction, but the form of the bunches changes with the drift direction. The bunches are straight with step-up drift (Fig. 2(a)) and wander with step-down drift (Fig. 2(b)). With a strong repulsive interaction, $A=300$, the step bunching is suppressed and the in-phase step wandering occurs. We have not studied the growth laws of the step width and the period of grooves, but the form of the grooves (Fig. 3) qualitatively agrees with the solution [14, 15] of Eq. (14). 


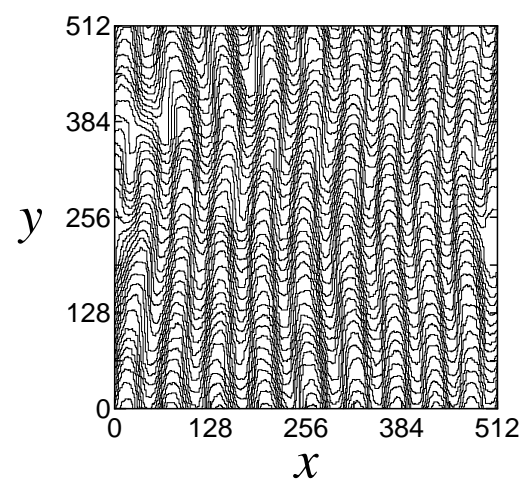

FIG. 3: Snapshot of step wandering induced by step-down drift with a strong step-step repulsion for $D_{1}>D_{2}$. Except for the repulsive potential, the parameters are the same as in Fig. 2(b).

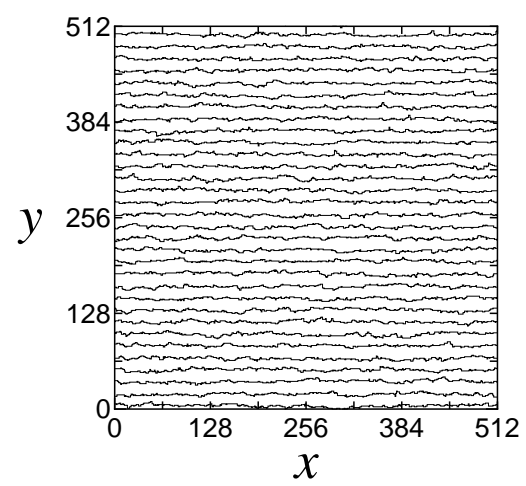

(a)

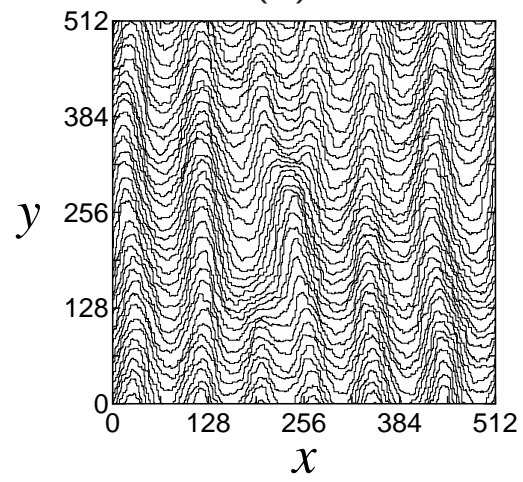

(b)

FIG. 4: Snapshots of a vicinal face for $D_{1}<D_{2}$ : with (a) stepup drift and (b) step-down drift. The repulsive interaction is absent.

We also carry out the simulation with $D_{1}<D_{2}$ (Fig. (4). The diffusion coefficients are $\left(D_{1}, D_{2}\right)=(0.1,1)$ and the step-step repulsion is neglected $(A=0)$. Other parameters are the same as Fig. 2 With step-up drift
(Fig. 4(a)), neither step wandering nor step bunching occurs. With step-down drift, the step wandering occurs, but no indication of bunching is seen (Fig. 4 (b)). As time passes, periodicity selection proceeds, resulting in straight grooves as those of Fig. 3.

\section{SUMMARY AND DISCUSSION}

In this paper, we studied the drift-induced morphological instabilities on a vicinal face with two phases. As is seen from Eq. (15), the step wandering occurs with step-down drift unless $D_{1}=D_{2}$. If $D_{1}=D_{2}$, our model reduces to a model of a simple vicinal face. We have already found 10] that, with step-down drift, wandering instability occurs in such a simple vicinal face if the kinetic coefficient of the step is finite. Although in the present paper we have assumed an infinite kinetic coefficient (local equilibrium at the steps), it must be finite in reality. Therefore we may say wandering instability is expected irrespective of the diffusion ratio. In all cases straight grooves parallel to the drift is produced due to the in-phase step wandering in Monte Carlo simulation if step bunching is suppressed.

In the $\mathrm{Si}(111)$ vicinal face near the transition temperature, the diffusion coefficient in the lower side of a step is larger than that in the upper side [18]. With the assumption that the ratio of the widths of the two structures is constant, the step bunching occurs irrespective of the current direction and the step wandering occurs with step-down current. In a vicinal face of large inclination, however, the step bunching is suppressed due to the strong step-step repulsion, and only grooves induced by the step wandering may be observed with step-down current.

In our model, we assumed that the boundary of the two structures moves in concert with the steps and the ratio of the widths of two structures is constant. The drift direction to cause the instabilities does not depend on the ratio of the widths. In reality the ratio of the widths changes with temperature 19] and the motion of the boundary does not automatically follow the steps. To study the morphological development of this system in detail, we need to extend our model to include the freedom of the motion of the boundary [20].

\section{Acknowledgments}

This work was supported by Grant-in-Aid for Scientific Research from Japan Society for the Promotion of Science. M. U. and Y. S. benefited from the inter-university cooperative research program of the Institute for Materials Research, Tohoku University. 
[1] M. Degawa, H. Nishimura, Y. Tanishiro, H. Minoda and K. Yagi, Jpn. J. Appl. Phys. 38 (1999) L308.

[2] M. Degawa, K. Thürmer, I. Morishima, H. Minoda, K. Yagi and E. D. Williamas, Surf. Sci. 493 (2001) 487.

[3] H. Minoda, J. Phys. Soc. Jpn. 71 (2002) 2944.

[4] J.-F. Nielsen, M. S. Pettersen and J. P. Pelz, Surf. Sci. 480 (2001) 84.

[5] S. Stoyanov, Jpn. J. Appl. Phys. 29 (1990) L659.

[6] M. Ichikawa and T. Doi, Appl. Phys. Lett. 60 (1992) 1082.

[7] J.-J. Métois, J.-C. Heyraud and A. Pimpinelli, Surf. Sci. 420 (1999) 250.

[8] M. Degawa, H. Minoda, Y. Tanishiro and K. Yagi, Surf. Sci. 461 (2000) L528.

[9] M. Sato, M. Uwaha and Y. Saito, Phys. Rev. B 62 (2000) 8452.

[10] M. Sato, M. Uwaha, Y. Saito and Y. Hirose, Phys. Rev. B 65 (2002) 245427.

[11] M. Sato, M. Uwaha, Y. Saito and Y. Hirose, Phys. Rev.
B 67 (2003) 125408.

[12] H. Hibino, Y. Homma, M. Uwaha and T. Ogino, Surf. Sci. 573 (2003) L222.

[13] R. Kato, M. Uwaha, Y. Saito and H. Hibino, Surf. Sci. 522 (2003) 64 .

[14] O. Pierre-Louis, C. Misbah, Y. Saito, J. Krug and P. Politi, Phys. Rev. Lett. 80 (1998) 4221.

[15] F. Gillet, O. Pierre-Louis, C. Misbah, Eur. Phys. J. B 18 (2000) 519 .

[16] P. Politi and C. Misbah, Phys. Rev. Lett. 92 (2004) 090601.

[17] Y. Saito, and M. Uwaha, Phys. Rev. B 49 (1994) 10677.

[18] H. Hibino, C.-W. Hu, T. Ogino and I. S. T. Tsong, Phys. Rev. B, 64 (2001) 245401.

[19] H. Yamaguchi and K. Yagi, Surf. Sci. 287/288 (1993) 820.

[20] R. Kato, M. Uwaha and Y. Saito, Surf. Sci. 550 (2004) 149. 